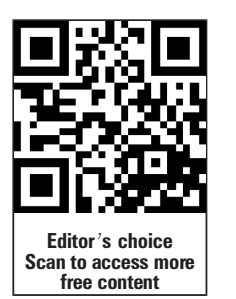

${ }^{1}$ Neuro Interventional Service, University of lowa, Carver College of Medicine, lowa City, lowa, USA

${ }^{2}$ Division of Interventional Neuroradiology, UCLA, Los Angeles, California, USA ${ }^{3}$ Department of Neurosurgery, UCLA, Los Angeles, California, USA

${ }^{4}$ UCLA Stroke Center, Los Angeles, California, USA ${ }^{5}$ Division of Diagnostic Neuroradiology, UCLA, Los Angeles, California, USA

Correspondence to Dr Michael T Froehler Department of Neurology, University of lowa Hospital, 200 Hawkins Drive, lowa City, IA 52242, USA;

michael-froehler@uiowa.edu

Received 15 February 2012 Revised 20 March 2012 Accepted 22 March 2012

Published Online First 22 May 2012

\title{
The hyperdense vessel sign on CT predicts successful recanalization with the Merci device in acute ischemic stroke
}

\author{
Michael T Froehler, ${ }^{1}$ Satoshi Tateshima, ${ }^{2}$ Gary Duckwiler, ${ }^{2}$ Reza Jahan, ${ }^{2}$ \\ Nestor Gonzalez, ${ }^{2,3}$ Fernando Vinuela, ${ }^{2}$ David Liebeskind, ${ }^{4}$ Jeffrey L Saver, ${ }^{3}$ \\ J Pablo Villablanca, ${ }^{5}$ For the UCLA Stroke Investigators
}

\begin{abstract}
Background The success of mechanical clot retrieval for acute ischemic stroke may be influenced by the characteristics of the occlusive thrombus. The thrombus can be partly characterized by CT, as the hyperdense vessel sign (HVS) suggests erythrocyte-rich clot whereas fibrin-rich clot may be isodense. We hypothesized that the physical clot characteristics that determine CT density may also determine likelihood of retrieval with the Merci device.
\end{abstract}

Methods We reviewed all acute stroke cases initially imaged with non-contrast CT before attempted Merci clot retrieval at a single center between 2004 and 2010. Each CT was blindly assessed for the presence or absence of the HVS, and post-retrieval angiograms were blindly assessed for reperfusion using the TICI scale.

Results 0 67 patients analyzed (mean age 69; median NIHSS 19; $61 \%$ female), the HVS was seen in 42, and no HVS was present in 25. Successful recanalization was achieved in $79 \%$ of patients with the HVS (33/42), but in only $36 \%(9 / 25)$ of patients without HVS ( $p=0.001)$. The HVS was the only significant predictor of recanalization while accounting for age, treatment with IV-tPA, clot location, stroke etiology, time to treatment, and number of retrieval attempts.

Conclusion The HVS in acute ischemic stroke was strongly predictive of successful recanalization using the Merci device. The HVS may indicate thrombi that are less adhesive compared with isodense clots that are more resistant to mechanical retrieval. The absence of HVS on pre-treatment CT may thus suggest the need for a more aggressive or alternative therapeutic approach.

\section{INTRODUCTION}

The emergent treatment of acute ischemic stroke is focused on revascularization. Thrombolysis with tPA has been used in the USA for approximately 15 years, ${ }^{1}$ and more recent efforts have shown the effectiveness of recanalization achieved by endovascular methods. ${ }^{2-4}$ However, not all patients experience successful recanalization with endovascular treatment, and those patients who fail to recanalize with attempted therapy do not benefit clinically. $^{5-8}$ The thrombectomy devices that are currently available in the USA can achieve successful recanalization in approximately $70 \%$ to $82 \%$ of acute stroke patients. ${ }^{4} 8$ While there are many technical and anatomic factors that may affect the ability to recanalize a clot, ${ }^{9}$ the physical characteristics of the occlusive thrombus itself may also impact the likelihood of successful mechanical recanalization. Therefore, it might be beneficial to ascertain information regarding clot characteristics prior to thrombectomy in the setting of acute ischemic stroke.

Some information regarding clot composition can be obtained from non-contrast head CT. In particular, the hyperdense vessel sign (HVS) on CT is an early sign that corresponds to the occlusive thrombus within the affected vessel (figure 1A). ${ }^{10} 11$ While specific, the HVS is not highly sensitive, as not all acute thrombi are hyperdense on CT. This may relate to varying composition of occlusive thrombi. Specifically, erythrocyte-rich clot has higher density on CT, and more often results in the HVS compared to thrombus with lower erythrocyte content. $^{12}$

The HVS may indicate the composition and the physical characteristics of the thrombus. Specifically, clot with a high erythrocyte content is hyperdense on CT, whereas fibrin-rich clot may be isodense. $^{13}$ Furthermore, CT-isodense clot with high fibrin content and lower relative erythrocyte content may be more firm and adhesive, ${ }^{14}$ potentially making it more difficult to retrieve by endovascular techniques. Ideally, the interventional strategy should take into account information about the physical characteristics of the clot that could be ascertained during non-invasive imaging before treatment.

The Merci device (Concentric Medical, Mountain View, California, USA) is a corkscrew-like mechanical clot retriever that is intended to ensnare the clot and remove it en bloc. We hypothesized that the physical clot characteristics that determine CT density may also determine likelihood of retrieval with the Merci device in the setting of acute ischemic stroke.

\section{METHODS \\ Patient population}

We retrospectively reviewed our institutional stroke database to identify all ischemic stroke patients that underwent non-contrast CT of the brain prior to attempted thrombectomy with the Merci clot retrieval device. Patient data was de-identified and the following clinical information was collected: 
Figure 1 (A) An example of the hypderdense vessel sign (HVS) in the right middle cerebral artery seen on non-contrast CT. The arrow indicates the clot seen in the proximal portion of the middle cerebral artery, but additional clot can also be seen as hyperdensity near the M1-M2 junction on this scan. (B) Patients that exhibited the HVS had a $79 \%$ likelihood of successful recanalization (right side). In contrast, when the HVS was not observed (isodense vessel; left side), mechanical recanalization was much more likely to fail. Only $36 \%$ of patients without the HVS experienced successful recanalization.
A

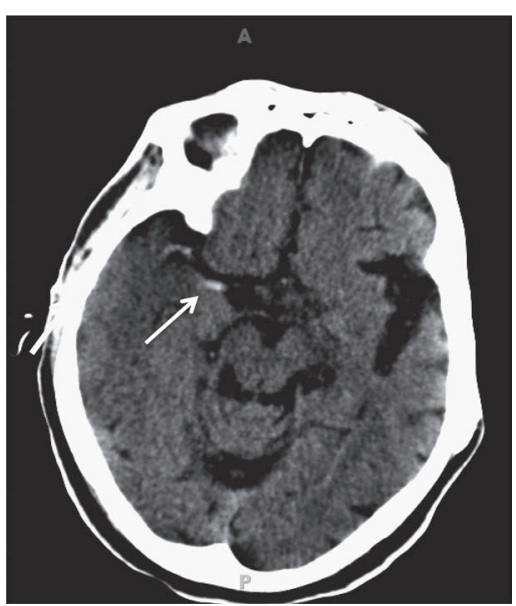

B

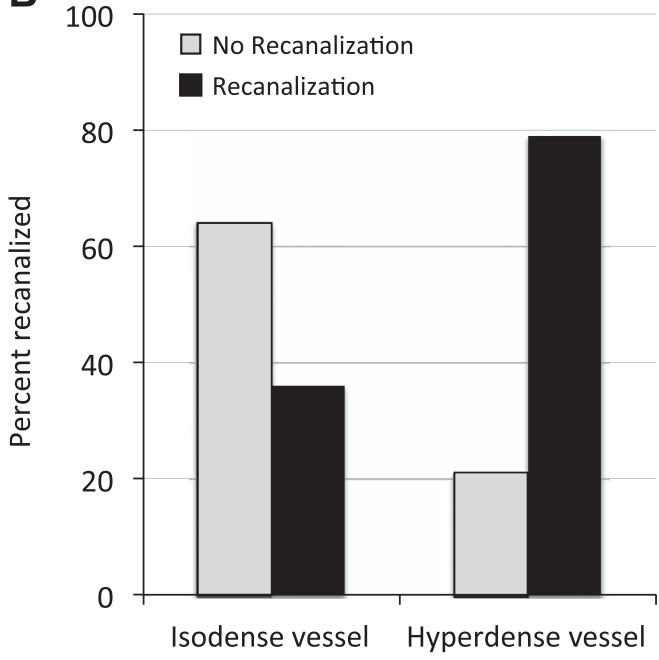

age, NIHSS, gender, treatment with IV tPA, clot location, stroke etiology, time to start of treatment since onset, and number of treatment attempts (pulls) with the Merci retriever. Any patient that received IV tPA was treated within $3 \mathrm{~h}$ of symptom onset, and all received the full dose of $0.9 \mathrm{mg} / \mathrm{kg}$ infused over $1 \mathrm{~h}$. Clot location was defined as the most proximal position of the thrombus; thus, a clot extending from ICA to MCA was characterized as an ICA clot, and all MCA occlusions involved the M1 segment. Stroke etiology was defined using a modified TOAST scheme ${ }^{15}$ to address large vessel occlusions treatable by endovascular means, and was categorized as cardioembolic, extracranial arterial disease, intracranial arterial disease, or cryptogenic. Time to treatment was defined as the number of minutes between the time the patient was last known well and the time of groin puncture.

\section{Neuroimaging}

Institutional non-contrast CT scans were performed using Siemens Somatom Sensation 16 or 64 detector series scanners (Siemens Medical Solutions, Erlangen, Germany). Scan images were obtained in a sequential manner using a field-of-view of $23 \mathrm{~cm}$, a kilovoltage ( $\mathrm{kVp}$ ) of 120 , effective mAs of $250-300$, slice collimation of $1.2 \mathrm{~mm}$ with $4.8 \mathrm{~mm}$ effective slice thickness, and a reconstruction kernel of $\mathrm{H} 40$ s medium for brain window and $\mathrm{H} 60$ for bone window. Image review was using a window setting of approximately 100, and a level of 40 .

All non-contrast CT scans were individually reviewed by an experienced neuroradiologist (JPV), who was blinded to results of the thrombectomy procedure. The neuroradiologist made a qualitative determination as to the presence or absence of the HVS in the region of the suspected thrombus (figure 1A). Additionally, for all scans that were performed at our institution, the density of the lesion was taken as the mean of three small circular or oval regions of interest (ROI) measurements placed entirely within the target and assessed in Hounsfield Units (HU). Using a similar technique, the density of a control artery was taken as the mean of three HU measurements of an uninvolved artery. A density ratio of the individual means was then calculated as affected artery density / control artery density. CT scans performed at other hospitals consisted of scans uploaded into our PACS imaging system and did not contain extractable HU density information, which precluded density measurements and inclusion in the density ratio analysis. Patients were also excluded from analysis if extensive atherosclerotic calcification within the target artery created artifact that prevented a reliable measurement of the density of the occlusive lesion.

All angiograms were individually reviewed by a neuro interventionalist (MTF), who was blinded to the results of the CT scan and the presence or absence of the HVS. The post-thrombectomy angiograms were assessed based on the Thrombolysis in Cerebral Infarction scale (TICI). ${ }^{16}$ Successful recanalization was defined as TICI 2 a or better, which corresponds to at least partial reperfusion with contrast passing beyond the site of initial occlusion. In cases where multiple devices were used, the angiogram after Merci thrombectomy was assessed rather than the final angiogram. In no case was another endovascular treatment attempted prior to Merci thrombectomy.

\section{Endovascular procedures}

All clot retrieval procedures were performed by neuro interventionalists experienced with the Merci clot retrieval system. In each case the entire Merci retrieval system was used, consisting of the Concentric $18 \mathrm{~L}$ microcatheter, the Merci device, and the Concentric balloon guide catheter, and was used according to the manufacturer's instructions for use. Treatment began with the placement of an eight or nine French Concentric balloon guide catheter in the cervical internal carotid artery, followed by navigation of the Concentric $18 \mathrm{~L}$ microcatheter over an 0.014" microguidewire across the lesion. The wire was removed and then a superselective angiogram was performed distal to the lesion. Next the Merci device was passed through the microcatheter and deployed in the distal portion of the thrombus. During the period of study, several generations of the Merci device were used, including the L, X, and V series. After the device was deployed, the balloon on the guide catheter was inflated to occlude proximal blood flow in the internal carotid artery. The Merci device was then slowly pulled into the guide catheter during aspiration through the guide catheter. Some cases also utilized the Concentric Distal Access Catheter as an intermediate catheter between the balloon guide catheter and microcatheter, for distal support. After retrieval of the device, the balloon was deflated and an angiogram was performed. If clot retrieval was unsuccessful, the above steps were repeated up to a maximum of six total thrombectomy attempts. In no case was intra-arterial tPA given prior to Merci thrombectomy.

\section{Statistical analysis}

Statistical analyses were carried out using SPSS (IBM, Armonk, New York, USA) on a Windows workstation. Parametric 
comparisons were made using Student t test, and non-parametric comparisons were made utilizing the chi-square or Fisher's exact test. A multivariate linear regression approach was used to assess recanalization based on age, IV tPA, clot location, stroke etiology, time to treatment, number of attempts, and presence of the HVS.

\section{RESULTS}

\section{Clinical characteristics}

We identified 68 patients who had a non-contrast head CT prior to undergoing Merci clot retrieval during the study period. One patient had calcific material in the cerebral arteries (density $>100 \mathrm{HU}$ ), and was therefore excluded from further analysis. Of the remaining 67 patients that were analyzed, $61.2 \%$ were female, the mean age was 69.3, and the median NIHSS was 19. Presence or absence of the HVS was defined by the blinded neuroradiologist's qualitative interpretation. The HVS was observed in 42 (63\%), and no HVS was seen in $25(37 \%)$. Consistent with the qualitative HVS assessment, the mean density ratio in the isodense group was $1.03 \pm 0.023$, and in the HVS group was $1.46 \pm 0.046$ ( $p<0.001$, t test). There was no difference in baseline clinical characteristics for the 67 patients analyzed (table 1). Mean time to treatment was $455 \mathrm{~min}$ in the isodense group and 394 min in the HVS group ( $p=0.341$, $t$ test), $32 \%$ of the isodense group and $36 \%$ of the HVS group received IV tPA ( $p=0.797$, Fisher's exact test), and the average number of thrombectomy attempts was 2.52 in the isodense group and 2.88 in the HVS group ( $p=0.361$, ANOVA). Stroke etiology did not differ between the groups ( $p=0.0625$; Fisher's exact test), with $80 \%$ cardioembolic in the isodense group and $69 \%$ in the HVS group, $4 \%$ extracranial arterial disease in the isodense group and $24 \%$ in the HVS group, $4 \%$ intracranial disease in the isodense group and none in the HVS group, and $12 \%$ cryptogenic in the isodense group and 7\% cryptogenic in the HVS group. There was a trend toward more MCA lesions in the isodense group and more ICA and basilar lesions in the hyperdense group, though this difference was not significant $(p=0.0686$; Fisher's exact test).

\section{Recanalization and the HVS}

Successful recanalization was achieved in $79 \%$ (33/42) of patients exhibiting the HVS compared with only $36 \%(9 / 25)$ of

Table 1 Clinical characteristics

\begin{tabular}{lllr}
\hline Characteristic & $\begin{array}{l}\text { Isodense } \\
(\mathbf{n}=\mathbf{2 5})\end{array}$ & $\begin{array}{l}\text { Hyperdense } \\
(\mathbf{n = 4 2 )}\end{array}$ & p Value \\
\hline NIHSS & 18.4 & 19.9 & 0.379 \\
Age & 73 & 67 & 0.186 \\
Female & $68 \%(17 / 25)$ & $57 \%(24 / 42)$ & 0.268 \\
Received IV tPA & $32 \%(8 / 25)$ & $(36 \%) 15 / 42$ & 0.797 \\
Clot location & & & 0.069 \\
$\quad$ MCA & $80 \%(20 / 25)$ & $55 \%(23 / 42)$ & \\
$\quad$ ICA & $20 \%(5 / 25)$ & $36 \%(15 / 42)$ & \\
Basilar & $0 \%(0 / 25)$ & $10 \%(4 / 42)$ & \\
CT density ratio & 1.03 & 1.46 & $<0.001$ \\
Stroke etiology & & & 0.063 \\
$\quad$ Cardioembolic & $80 \%(20 / 25)$ & $69 \%(29 / 42)$ & \\
$\quad$ Extracranial arterial disease & $4 \%(1 / 25)$ & $24 \%(10 / 42)$ & \\
$\quad$ Intracranial arterial disease & $4 \%(1 / 25)$ & $0 \%(0 / 42)$ & \\
$\quad$ Cryptogenic & $12 \%(3 / 25)$ & $7 \%(3 / 42)$ & \\
Time to treatment (mean \pm SEM) & $455.2 \pm 53.1$ & $394.1 \pm 37.6$ & 0.341 \\
Number of attempts (mean \pm SEM) & $2.52 \pm 0.64$ & $2.88 \pm 0.48$ & 0.361 \\
Successful recanalization & $36 \%(9 / 25)$ & $79 \%(33 / 42)$ & 0.001 \\
\hline
\end{tabular}

Table 2 Isodense group

\begin{tabular}{llll}
\hline & $\begin{array}{l}\text { No Recanalization } \\
(\mathbf{n = 1 6 )}\end{array}$ & $\begin{array}{l}\text { Recanalized } \\
(\mathbf{n}=\mathbf{9})\end{array}$ & $\mathbf{p}$ Value \\
\hline $\begin{array}{l}\text { Received IV tPA } \\
\text { Stroke etiology }\end{array}$ & $31 \%(5 / 16)$ & $33 \%(3 / 9)$ & 0.999 \\
$\quad$ Cardioembolic & $75 \%(12 / 16)$ & $89 \%(8 / 9)$ & 0.999 \\
$\quad$ Extracranial arterial disease & $6 \%(1 / 16)$ & $0 \%(0 / 9)$ & \\
Intracranial arterial disease & $6 \%(1 / 16)$ & $0 \%(0 / 9)$ & \\
$\quad$ Cryptogenic & $13 \%(2 / 16)$ & $11 \%(1 / 9)$ & \\
Time to treatment (mean \pm SEM) & $462.1 \pm 66.3$ & $442.5 \pm 97.2$ & 0.867 \\
& $(\mathrm{n}=11)$ & $(\mathrm{n}=6)$ & \\
Number of attempts (mean \pm SEM) & $2.88 \pm 0.43$ & $1.89 \pm 0.35$ & 0.131 \\
\hline
\end{tabular}

patients without the HVS (figure 1B). This difference was highly significant by $\chi^{2}$ comparison and Fisher's exact test $(p=0.001)$.

A multivariate regression analysis of recanalization was carried out for the following factors: age, treatment with IV tPA, clot location, stroke etiology, time to treatment, number of attempts, and presence of the HVS. Of these variables, only the HVS was predictive of recanalization ( $p=0.004$; multivariate linear regression). Recanalization was not significantly associated with clot location $(p=0.163)$ or use of IV tPA $(p=0.124)$ in multivariate analysis.

\section{Within-groups analysis}

Within the isodense group (no HVS), there were no significant differences in the variables examined between the 9 recanalized and 16 non-recanalized patients (table 2). Of those without the HVS that were not recanalized, 31\% (5/16) received IV tPA, compared to $33 \%(3 / 9)$ that were recanalized ( $p=0.999$, Fisher's exact). Etiology also did not differ between recanalized and nonrecanalized groups ( $p=0.999$, Fisher's exact). The mean time to treatment was $462.1 \mathrm{~min} \pm 66.3$ in the non-recanalized group $(n=11)$ and $442.5 \mathrm{~min} \pm 97.2(n=6)$ in the recanalized group $(\mathrm{p}=0.867$, $\mathrm{t}$ test). Mean number of treatment attempts was $2.88 \pm 0.43$ in the non-recanalized group and $1.89 \pm 0.35$ in the recanalized group $(p=0.131$, one-way ANOVA).

Within the HVS group, there were also no significant differences between the 33 patients that were recanalized and the 9 that were not recanalized (table 3). Of those with the HVS that were not recanalized, 55\% (5/9) received IV tPA, compared to $30 \%(10 / 33)$ that were recanalized $(p=0.2417$, Fisher's exact). Etiology did not differ between the recanalized and non-recanalized groups $(p=0.0936$, Fisher's exact), though there were more patients with extracranial arterial disease with the HVS, all of whom were recanalized (10/10). Mean time to treatment was $298.0 \mathrm{~min} \pm 14.9(\mathrm{n}=4)$ in the non-recanalized group and $393.6 \pm 47.5(n=19)$ in the recanalized group ( $p=0.375$, t-test). While there was a trend toward shorter time to treatment in the non-recanalized group, the difference was not significant due to

Table 3 Hyperdense group

\begin{tabular}{llll}
\hline & $\begin{array}{l}\text { No Recanalization } \\
(\mathbf{n}=\mathbf{9})\end{array}$ & $\begin{array}{l}\text { Recanalized } \\
(\mathbf{n}=\mathbf{3 3})\end{array}$ & $\mathbf{p ~ V a l u e}$ \\
\hline $\begin{array}{l}\text { Received IV tPA } \\
\text { Stroke etiology }\end{array} \quad 56 \%(5 / 9)$ & $30 \%(10 / 33)$ & 0.242 \\
$\quad$ Cardioembolic & $89 \%(8 / 9)$ & $64 \%(21 / 33)$ & 0.094 \\
$\quad$ Extracranial arterial disease & $0 \%(0 / 9)$ & $30 \%(10 / 33)$ & \\
$\quad$ Intracranial arterial disease & $0 \%(0 / 9)$ & $0 \%(0 / 33)$ & \\
$\quad$ Cryptogenic & $11 \%(1 / 9)$ & $6 \%(1 / 33)$ & \\
Time to treatment (mean \pm SEM) & $298.0 \pm 14.9$ & $393.6 \pm 47.5$ & 0.375 \\
& $(\mathrm{n}=4)$ & $(\mathrm{n}=19)$ & \\
Number of attempts (mean \pm SEM) & $2.89 \pm 0.56$ & $2.88 \pm 0.27$ & 0.986 \\
\hline
\end{tabular}


the low sample size in the non-recanalized group, as time to treatment data were not available for all patients. Mean number of treatment attempts was $2.89 \pm 0.56$ in the non-recanalized group and $2.88 \pm 0.27$ in the recanalized group $(p=0.986$, oneway ANOVA).

\section{DISCUSSION}

In the present series, acute ischemic stroke patients exhibiting the HVS had a much higher chance of successful recanalization with the Merci device compared to patients without the HVS on noncontrast head CT. In multivariate analyses, this statistically significant difference persisted after accounting for use of IV tPA, clot location, stroke etiology, time to treatment, and number of thrombectomy attempts. This finding has important implications regarding the endovascular approach to thrombectomy or thrombolysis, and the composition of the thrombus causing ischemic stroke.

Imaging characteristics of clot may reflect the composition of the thrombus. Recently, Liebeskind et al performed histologic assessment of clots retrieved from patients with acute ischemic stroke, and found a significantly higher $(p=0.016)$ erythrocyte content in clots with the HVS (47\%) compared to isodense clots $(22 \%) .{ }^{13}$ Furthermore, they report that only $20 \%$ of fibrin-rich thrombi were associated with the HVS. These data suggest that hyperdense clot has a higher erythrocyte content, whereas isodense clot may be more fibrin rich. This relationship has also been observed in vitro, where CT density was demonstrated to increase directly in proportion to hematocrit. ${ }^{12}$ Thus, hyperdense clot on CT suggests an erythrocyte-rich thrombus.

It is interesting to speculate that the composition of thrombus may be related to stroke etiology. However, Liebeskind et al found no relationship between etiology and thrombus histopathology, ${ }^{13}$ and a previous analysis of thromboemboli retrieved from stroke patients showed similar pathology regardless of source. ${ }^{17}$ One group did recently observe higher mechanical recanalization rates in patients with stroke due to proximal carotid occlusion compared to cardioembolic or cryptogenic stroke, though no histopathological analysis was included in that study. ${ }^{18}$ In our current series, there was a higher number of strokes due to extracranial arterial disease in the HVS group, though this difference was not statistically significant. It might be that larger studies will reveal a tendency toward higher erythrocyte content in thrombi that arise from large artery atherosclerosis.

The relative proportion of erythrocyte and fibrin in thrombus composition may have important influences on the mechanical properties of the clot. Gersh et al have studied the influence of erythrocyte concentration on clot structural and mechanical properties in vitro. ${ }^{14}$ They found that higher levels of erythrocytes causes heterogeneity of clot composition, disrupts fibrin structure, and results in increased viscosity and decreased elasticity of the clot. These influences on the physical properties of clot may have important implications for endovascular therapy, including mechanical clot retrieval.

Our current study has shown that hyperdense clot is more likely to respond to Merci mechanical thrombectomy than isodense clot. Others have shown also that hyperdense clot has a higher erythrocyte concentration, ${ }^{12}{ }^{13}$ which in turn suggests increased viscosity and deformability. ${ }^{14}$ It may be these physical characteristics-specifically viscosity and deformability-that lead to increased efficacy of the Merci device. In contrast, isodense clot is associated with higher fibrin content, which suggests increased elasticity ${ }^{14}$ and stiffness. ${ }^{19}$ These characteristics-increased elasticity and stiffness-may decrease effectiveness of the Merci device.
The physical composition of the clot may also predict response to pharmacologic thrombolysis. Liebeskind et al ${ }^{13}$ have shown that erythrocyte-rich thrombi are associated with increased CT density and with increased susceptibility on T2*weighted gradient echo MRI. Kimura et al have shown that T2* susceptible, and therefore erythrocyte-rich, clots are unlikely to recanalize with intravenous $\mathrm{tPA}^{20}$ In contrast, clot that is not associated with $\mathrm{T} 2 *$ susceptibility, and therefore fibrin-rich, does respond to tPA. ${ }^{20} 21$ Similarly, others have shown that intraarterial thrombolysis appears to be more successful than intravenous tPA in patients with the HVS. ${ }^{22}$ Thus, fibrin-rich clots may respond better to tPA than erythrocyte-rich clots.

These observations, in combination with our current results, suggest that the absence of the HVS implies a fibrin-rich $\operatorname{clot}^{13}$ that may respond poorly to mechanical retrieval, but is more likely to recanalize with tPA. ${ }^{20}$ Therefore, appropriate endovascular rescue therapy for patients without the HVS after initial failure of mechanical thrombectomy may include the use of intra-arterial thrombolytics rather than continued attempts at mechanical therapy alone.

These results make it clear that different composition and characteristics of thrombi lead to varying response to treatment. It is therefore important to develop models of different thrombus types so that thrombolysis and thrombectomy methods can be studied quantitatively. Chueh et al have recently reported the mechanical characterization of different thrombotic embolus analogs of different stiffness and elasticity. ${ }^{19}$ Likewise, Kan et al have described a new animal stroke model utilizing a fibrin-rich clot that displays much greater elasticity compared to standard, erythrocyte-rich clot. ${ }^{23}$ The laboratory investigation of thrombolytics and thrombectomy devices utilizing different thrombus analogs will enable the characterization of different clot types and their ideal treatment methods. This may also foster the development of novel thrombectomy devices designed specifically for more resistant clot types, such as the fibrin-rich thrombi that seem less likely to recanalize with the Merci device. Studying the imaging characteristics of these thrombi will allow the early, non-invasive identification of thrombus composition and inferred characterization of the physical properties of the clot, which will support informed, real-time decision making regarding endovascular treatment for acute ischemic stroke.

We have shown that hyperdense, erythrocyte-rich clot responds better to mechanical thrombectomy compared to isodense, fibrin-rich clot. Others have shown that fibrin-rich $\operatorname{clot}^{13}$ is more likely to respond to pharmacologic thrombolysis with tPA. ${ }^{20}$ Therefore, in patients presenting with large vessel occlusion, the presence of the HVS on CT may predict a favorable response to mechanical thrombectomy. In contrast, the absence of the HVS may serve as an indicator of adhesive clot that may require a more aggressive or alternative endovascular approach, potentially including the use of intra-arterial pharmacologic thrombolytics.

Acknowledgments We are grateful to Dr Minako Hayakawa for her insightful review of the manuscript.

Contributors MTF was primarily responsible for study design, statistical analysis, and composing the manuscript, and is gaurantor of the work. ST and JPV participated in study design, data collection, and composition of the work. GD, RJ, NG, FV, DL, and JS were involved in data collection and editing of the manuscript.

Funding Dr Froehler was supported by the A P Giannini Foundation. Dr Gonzalez was supported by the Ruth and Raymond Stotter Endowement. Dr Liebeskind receives funding from the $\mathrm{NIH}$, including grants K24 NS072272-01, K23 NS054084-05, P50 NS044378-06. Dr. Villablanca receives funding from the NIH, including grants U01 NS044364, P50 NS044378, and from the NINDS U01NS052220. 
Competing interests Drs Tateshima, Duckwiler, Jahan, Gonzalez, Vinuela, Liebeskind, Saver, and Villablanca are employees of the University of California which holds patent rights in retrieval devices for stroke. Dr. Tateshima has received honoraria from Concentric Medical, the maker of the Merci device, and from Century Medical, the Japanese distributor of the Merci device. Drs. Duckwiler and Liebeskind serve as scientific advisors for Concentric Medical.

Ethics approval Ethics approval statement was approved by UCLA IRB.

Provenance and peer review Not commissioned; externally peer reviewed.

\section{REFERENCES}

1. Anon. Tissue plasminogen activator for acute ischemic stroke. The National Institute of Neurological Disorders and Stroke rt-PA Stroke Study Group. N Engl J Med 1995;333:1581-7

2. Smith WS, Sung G, Starkman S, et al. Safety and efficacy of mechanical embolectomy in acute ischemic stroke: results of the MERCI trial. Stroke 2005;36:1432-8.

3. Furlan A, Higashida R, Wechsler L, et al. Intra-arterial prourokinase for acute ischemic stroke. The PROACT II study: a randomized controlled trial. Prolyse in Acute Cerebral Thromboembolism. JAMA 1999;282:2003-11.

4. The penumbra pivotal stroke trial: safety and effectiveness of a new generation of mechanical devices for clot removal in intracranial large vessel occlusive disease. Stroke 2009; 40:2761-8.

5. Rha JH, Saver JL. The impact of recanalization on ischemic stroke outcome: a metaanalysis. Stroke 2007;38:967-73.

6. Fields JD, Lutsep HL, Smith WS. Higher Degrees of recanalization after mechanical thrombectomy for acute stroke are associated with Improved outcome and decreased mortality: pooled analysis of the MERCI and Multi MERCI Trials. AJNR Am J Neuroradiol 2011:32:2170-4.

7. Nogueira RG, Liebeskind DS, Sung G, et al. Predictors of good clinical outcomes mortality, and successful revascularization in patients with acute ischemic stroke undergoing thrombectomy: pooled analysis of the Mechanical Embolus Removal in Cerebral Ischemia (MERCI) and Multi MERCI Trials. Stroke 2009;40:3777-83

8. Smith WS, Sung G, Saver J, et al. Mechanical thrombectomy for acute ischemic stroke: final results of the Multi MERCI trial. Stroke 2008;39:1205-12.

9. Gralla J, Brekenfeld C, Mordasini P, et al. Mechanical thrombolysis and stenting in acute ischemic stroke. Stroke 2012:43:280-5.
10. Gacs G, Fox AJ, Barnett $\mathrm{HJ}$, et al. CT visualization of intracranial arterial thromboembolism. Stroke 1983;14:756-62.

11. Pressman BD, Tourje EJ, Thompson JR. An early CT sign of ischemic infarction: increased density in a cerebral artery. AJR Am J Roentgenol 1987; 149:583-6

12. Kirchhof $\mathbf{K}$, Welzel T, Mecke $\mathrm{C}$, et al. Differentiation of white, mixed, and red thrombi: value of CT in estimation of the prognosis of thrombolysis phantom study. Radiology 2003;228:126-30.

13. Liebeskind DS, Sanossian N, Yong WH, et al. CT and MRI early vessel signs reflect clot composition in acute stroke. Stroke 2011;42:1237-43.

14. Gersh KC, Nagaswami C, Weisel JW. Fibrin network structure and clot mechanica properties are altered by incorporation of erythrocytes. Thromb Haemost 2009;102:1169-75

15. Adams HP Jr, Bendixen BH, Kappelle LJ, et al. Classification of subtype of acute ischemic stroke. Definitions for use in a multicenter clinical trial. TOAST. Trial of Org 10172 in Acute Stroke Treatment. Stroke 1993;24:35-41.

16. Higashida RT, Furlan AJ, Roberts $\mathrm{H}$, et al. Trial design and reporting standards for intra-arterial cerebral thrombolysis for acute ischemic stroke. Stroke 2003;34: e109-37.

17. Marder VJ, Chute DJ, Starkman S, et al. Analysis of thrombi retrieved from cerebra arteries of patients with acute ischemic stroke. Stroke 2006;37:2086-93

18. Hussain MS, Lin R, Cheng-Ching E, et al. Endovascular treatment of carotid embolic occlusions has a higher recanalization rate compared with cardioembolic occlusions. J Neurointerv Surg 2010;2:71-3

19. Chueh JY, Wakhloo AK, Hendricks GH, et al. Mechanical characterization of thromboemboli in acute ischemic stroke and laboratory embolus analogs. AJNR Am J Neuroradiol 2011;32:1237-44

20. Kimura K, Iguchi $Y$, Shibazaki $K$, et al. M1 susceptibility vessel sign on T2* as a strong predictor for no early recanalization after IV-t-PA in acute ischemic stroke. Stroke 2009; 40:3130-2.

21. Kimura K, Sakamoto Y, Aoki J, et al. Clinical and MRI predictors of no early recanalization within 1 hour after tissue-type plasminogen activator administration. Stroke 2011:42:3150-5

22. Mattle HP, Arnold M, Georgiadis D, et al. Comparison of intraarterial and intravenous thrombolysis for ischemic stroke with hyperdense middle cerebral artery sign. Stroke 2008:39:379-83.

23. Kan I, Yuki I, Murayama Y, et al. A novel method of thrombus preparation for use in a swine model for evaluation of thrombectomy devices. AJNR Am J Neuroradiol 2010:31:1741-3. 